\title{
CYSTIC TERATOMA OF MESENTERY: A CASE REPORT AND REVIEW OF LITERATURE
}

Shashikumar¹, Rajendra Kumar², Nanjaraj C. P33, Vishwanath Joshi ${ }^{4}$

\section{HOW TO CITE THIS ARTICLE:}

Shashi Kumar, Rajendra Kumar, Nanjaraj C. P, Vishwanath Joshi. “Cystic Teratoma of Mesentery: A Case Report and Review of Literature". Journal of Evolution of Medical and Dental Sciences 2014; Vol. 3, Issue 40,

September 01; Page: 10183-10187, DOI: $10.14260 /$ jemds/2014/3323

ABSTRACT: Teratomas are neoplasms arising from totipotent primordial cells and display a mixture of tissues of tri dermal or bi dermal origin, foreign to the anatomic site, in which they arise.[1,7] Teratomas are usually found in the sacrococcygeal area in children and gonads in adults. Less frequently they can be seen in sites such as neck, mediastinum, retro peritoneum, cranial cavity,

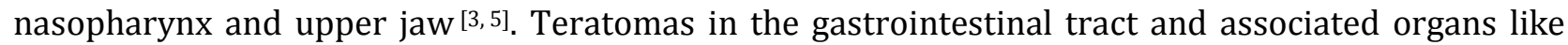
stomach and pancreas are rare, and there are only occasional case reports on mesenteric teratoma [3,5]. We report a case of cystic mesenteric teratoma in a 12 year old male patient with review of relevant literature.

KEYWORDS: Teratomas, Mesenteric, Totipotent, Calcifications.

INTRODUCTION: Mesenteric teratomas are uncommon, benign neoplasms that occur primarily in children.[2] Principle findings are a well-defined mass with separate cystic and solid components of varying proportions, discrete areas with densities similar to that of fat, or coarse, globular calcifications within the solid component. Recognition of these findings may allow the radiologist to make a correct preoperative diagnosis of teratoma ${ }^{[1]}$.

CASE REPORT: A 12 years old male presented with a 6 month history of lower abdominal distension. There was no history suggestive of altered bowel habits or urinary complaints. Per abdomen examination revealed a large, intra-abdominal, non-tender, and lump occupying the left side of abdomen.

There was no free fluid in the abdomen clinically. Ultrasonography revealed an intraabdominal cystic lesion predominantly in the left lumbar and umbilical regions (Fig. 1). The lesion had thick septations with area of floating hyper echoic elements s/o fat component. No evidence of calcifications was noted. There was no evidence of ascites or lymphadenopathy.

CT scan of the abdomen revealed a large well defined intra peritoneal predominantly cystic lesion in left lumbar and umbilical regions. The lesion was predominantly of water attenuation with thick incomplete non enhancing septa within (Fig. 2). Discrete area of fat density was present within the lesion (Fig. 3). The lesion was causing mass effect in the form of displacement of small bowel loops to the right side (Fig. 4).

A diagnosis of mesenteric teratoma was made based on imaging findings. At surgery the lesion was found to be within the jejunal mesentery (Fig. 5). Areas of fat and fluid were present. (Fig. 6) Histopathological findings of areas of adipocytes with lining of squamous epithelium of the cystic component confirmed the diagnosis of mature cystic teratoma of the mesentery. 
DISCUSSION: Teratoma of the mesentery is an uncommon benign neoplasm that occurs primarily in pediatric patients. The clinical presentation is usually non-specific. Abdominal discomfort may be present because, tumour causes compression on bladder and gastrointestinal tract [6].

Classically the teratoma originates in the midline position. But in the abdomen, it usually takes the position of one of the paravertebral gutters, perhaps due to its size and weight of the solid part of the constituent elements.

Diagnosis is done by imaging modalities and examination of histological specimen. ${ }^{44]}$ Ultrasound is useful and can help in the confirmation of the diagnosis only a few cases have been reported to make the diagnosis by ultrasound, preoperatively. ${ }^{[7]}$ CT scan is the modality of choice for diagnosis of teratomas because of its ability to detect different densities of mass.

On ultrasound the diagnosis of teratoma should be suspected if a cystic mass is detected with fat-fluid level and calcifications. Specific CT scan characteristic of mature mesenteric cystic teratoma is a well marginated mass with one or more cystic components of varying proportions, frequently processing areas of calcification or fat. Frequently a solid protuberance projects into the cyst cavity (Rokitansky protuberance, dermoid plug). Calcifications are commonly seen in the solid component or in the wall. On MRI, cystic teratomas reveal the signal characteristic of fat (hyper intense on T1weighted images) and water (hypo intense on T1 weighted images and hyper intense on T2-weighted images) chemical shift between the fatty and watery contents is a diagnostic finding at MR imaging. In addition to the detection of fat, gravity-dependent layering, palm tree-like protrusions (Rokitansky protuberance) and fat-fluid levels are other imaging characteristics of mature cystic teratomas [6].

Immature teratoma is characterized by the presence of elements that resemble embryonic tissues, including neuroglial or neuro-epithelial components that may coexist along with mature tissues. Immature elements are usually recognized as solid parts at CT and MR imaging. The existence of a solid component can be a sign of an immature teratoma, although mature teratomas sometimes have large solid parts (Rokitansky protuberances). In addition, immature teratomas without such prominent solid parts are occasionally seen. Therefore, distinguishing immature from mature teratomas on imaging is very difficult [7].

Pathologically, a cystic mesenteric teratoma is a well-defined mass with both cystic and solid components containing multiple tissue types. Histopathology is usually necessary to rule out changes of malignancy.[6]

The most common type of mesenteric cyst in pediatric population is lymphangioma. Mesenteric cystic lymphangioma may mimic cystic teratoma radiologically, but can be differentiated by its multiloculated appearance and absence of fat/ calcifications or bone within. Further differential diagnosis of single fat containing mesenteric mass will be liposarcoma and mesenteric lipodystrophy. However lipodystrophy is not cystic and liposarcoma usually does not contain fluid levels [6].

In summary, it's important to establish a pre-operative diagnosis of mesenteric teratomas, as these tumors are benign, thereby allowing surgical treatment. The radiologic diagnosis is suggested in most cases, particularly using cross sectional imaging techniques. The major role of the radiologist is to characterize these abdominal masses and their exact mesenteric origin.

\section{REFERENCES:}

1. Bowen B, Ros PR, McCarthy MJ, Olmsted WW, Hjermstad. Gastrointestinal teratomas: CT and US appearance with pathologic correlation. Radiology 1987; 162: 431-433. 
2. Stoupis C, Ros PR, Abbitt PL, Burton SS, Gauger J. Bubbles in the belly: imaging of cystic mesenteric or omental masses. Radiographics 1994 Jul; 14 (4): 729-37.

3. Chiba T, Iwami D, Kikuchi Y. Mesenteric teratoma in an 8- month-old girl. J Pediatr Surg 1995; 30: 120.

4. De J, Banerjee M, Biswas PK, Banerjee AK, Karim R. Mature teratoma of the mesentery. J Indian Med Assoc. 2002 Mar; 100 (3): 198-9.

5. Al-Arfaj AA, El-Shawarby MA, Al-Mulhim FA et al, Mesenteric cystic teratoma in children. Saudi Med J. 2003 Dec; 24 (12): 1388-90.

6. Hiral P P, Vasavada D P. A Mesenteric teratoma in an adult male - a rare case. Indian J Radiol Imaging 2004; 14: 257-9.

7. Raychaudhari C, Prajapati H, Shah H K. Two Cases of Immature Mesenteric Teratoma, Ind J Radiol Imag 2006 16:4:567-570.

FIG. 1: Sagittal USG showing a intrabdominal cystic lesion with thick septations with area of floating hyperechoic elements s/o fat component.

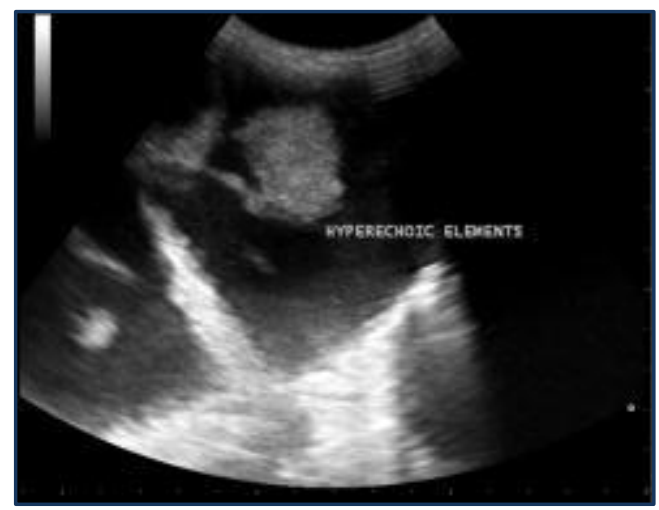

\section{FIG. 1}

Fig. 2: CT showing a well-defined intra peritoneal predominantly cystic lesion in left lumbar and umbilical regions. The lesion was predominantly of water attenuation with thick incomplete non enhancing septa within.

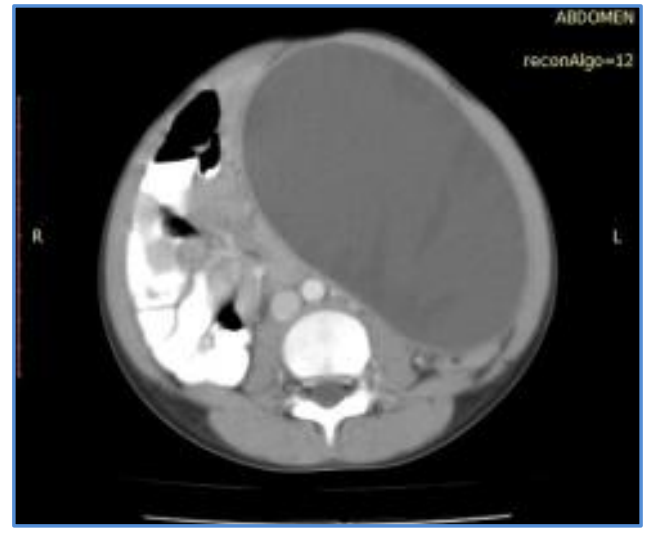

\section{FIG. 2}


Fig. 3: CT scan showing discrete area of fat density was present within the lesion.

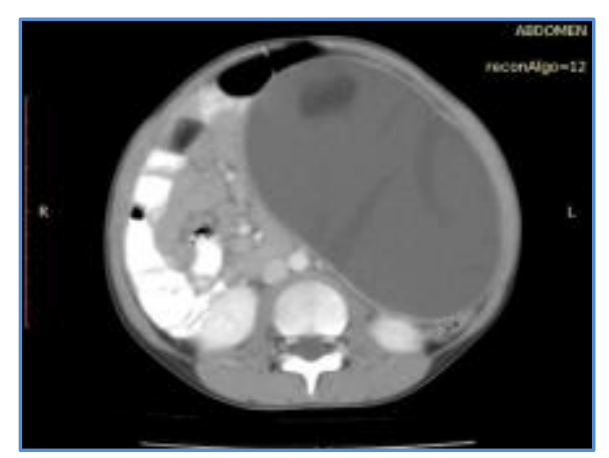

\section{FIG. 3}

Fig. 4: CT scan showing the cystic lesion causing mass effect in the form of displacement of small bowel loops to the right side.

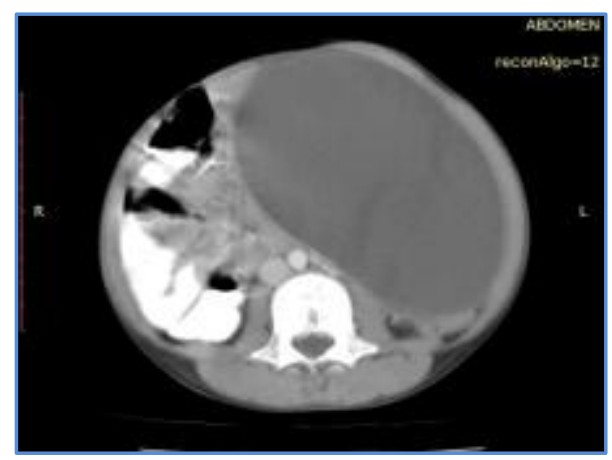

\section{FIG. 4}

Fig. 5: At surgery the lesion was found to be within the jejunal mesentery.

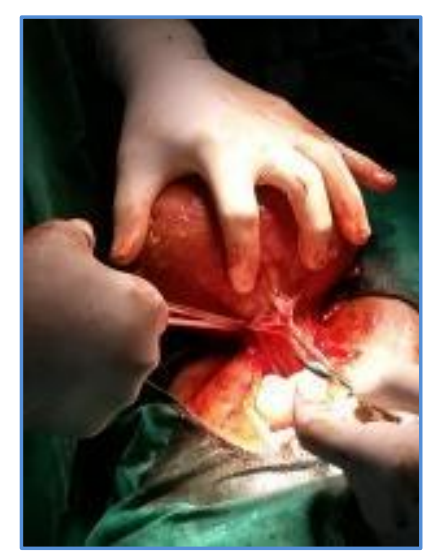

FIG. 5 


\section{CASE REPORT}

Fig. 6: Gross specimen of the lesion showing fat and cystic components.

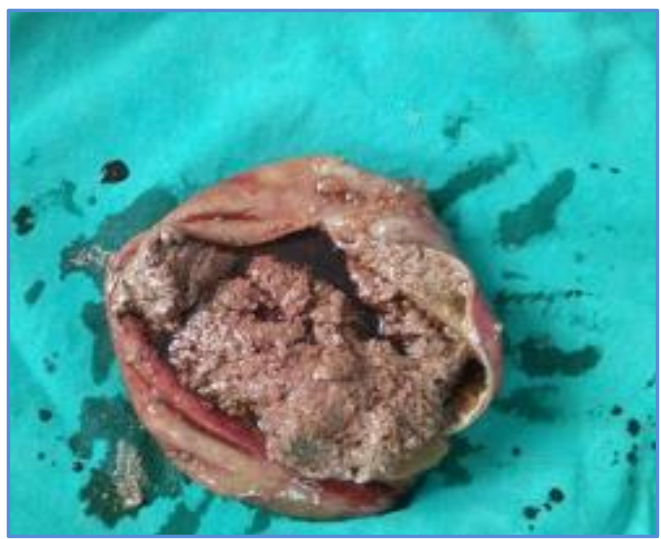

FIG. 6

\section{AUTHORS:}

1. Shashi kumar

2. Rajendra Kumar

3. Nanjaraj C. P.

4. Vishwanath Joshi

\section{PARTICULARS OF CONTRIBUTORS:}

1. Associate Professor, Department of Radiodiagnosis, Mysore Medical College and research Institute, Mysore, Karnataka.

2. Associate Professor, Department of Radiodiagnosis, Mysore Medical College and research Institute, Mysore, Karnataka.

3. Professor, Department of Radiodiagnosis, Mysore Medical College and research Institute, Mysore, Karnataka.
4. Post Graduate, Department of Radiodiagnosis, Mysore Medical College and research Institute, Mysore, Karnataka.

\section{NAME ADDRESS EMAIL ID OF THE CORRESPONDING AUTHOR:}

Dr. Shashikumar M.R., $241,1^{\text {st }}$ Stage,

Brindavana Extension, Mysore-570020.

Email: drmrshashikumar@gmail.com

Date of Submission: 12/08/2014. Date of Peer Review: 13/08/2014. Date of Acceptance: 25/08/2014. Date of Publishing: 01/09/2014. 